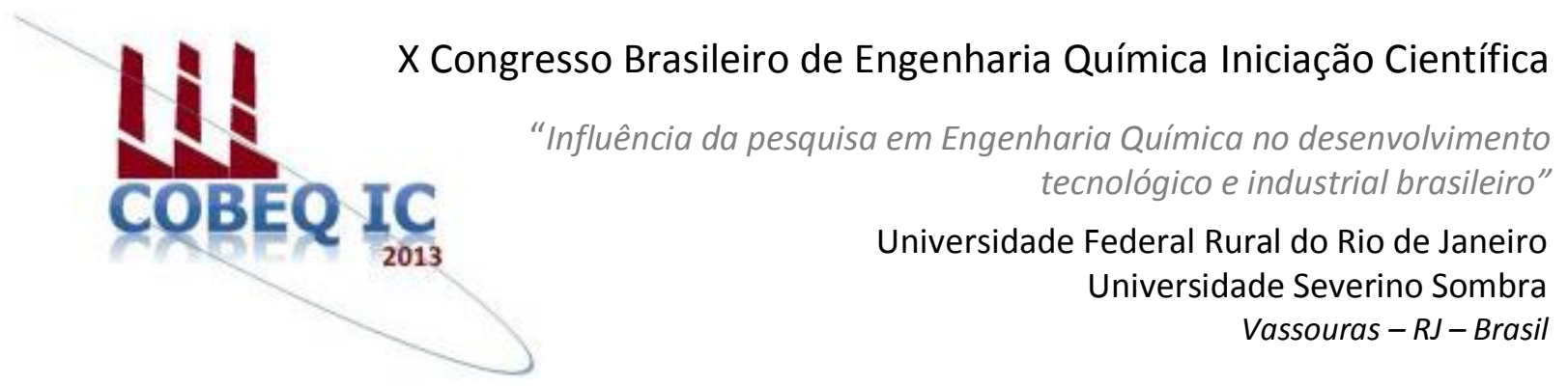

\title{
PRODUÇÃO DE ETANOL CELULÓSICO POR COMPLEXO ENZIMÁTICO DE FUNGO DO CERRADO: EFEITO DO MEIO SÓLIDO NA PRODUÇÃO DA ENZIMA E CARGA DE BAGAÇO NA FERMENTAÇÃO ETÍLICA
}

\author{
SOUSA $^{* 1}$, V. C.; GUEDES $^{2}$, K.; PLÁCIDO ${ }^{2}$, M.; LOPES, V.S. ${ }^{3}$; \\ FISCHER $^{3}$, J.; CARDOSO, V.L.; FILHO, U.C \\ (1) Aluna do Curso de Bacharelado em Engenharia Química UFU; \\ ${ }^{(2)}$ Bolsista de iniciação científica do Curso de Bacharelado em Engenharia Química UFU; \\ (3) Alunas do Curso de Pós-Graduação em Engenharia Química UFU \\ (4) Docentes - FEQUI/UFU \\ Endereço - Faculdade de Engenharia Química da Universidade Federal de Uberlândia. Av. João \\ Naves de Ávila, 2121, Bloco 1K, Campus Santa Mônica, Uberlândia - MG, CEP 38408-100, \\ e-mail: ucfilho@feq.ufu.br
}

RESUMO - Na produção de etanol de celulose, a geração de extrato enzimático bruto e a busca por novos microrganismos representam duas estratégias para reduzir o custo do processo. No presente trabalho foi feita a avaliação do potencial do fungo do cerrado, Chrysonilia sitophila, para a produção de etanol de segunda geração. A produção de complexos enzimáticos foi realizada com 12 meios de composições distintas, contendo farelo de arroz e bagaço de cana-de-açúcar explodido, e, uma vez selecionado o meio, foi feito o estudo do melhor tempo de produção de etanol, composição e carga. Foi observado que o meio composto de $1 / 3$ de bagaço de cana explodido e $2 / 3$ de farelo de arroz foi o que favoreceu a maior conversão de bagaço em etanol com geração de aproximadamente $5,3 \mathrm{~g} / \mathrm{L}$ de etanol. No estudo da fermentação etílica, foi observado que ocorreu um aumento significativo na produção de etanol, quando a concentração de bagaço foi aumentada de 190 para $200 \mathrm{~g} / \mathrm{L}$, e uma significativa diminuição na produção de etanol, quando a concentração de bagaço foi aumentada de 200 para $220 \mathrm{~g} / \mathrm{L}$.

Palavras chave: Chrysonilia sitophila, bagaço de cana-de-açúcar, celulose.

\section{INTRODUÇÃO}

O etanol é gerado desde o fim do século XIX, mas somente nos últimos 20 anos essa técnica se desenvolveu o suficiente para justificar seu uso de forma econômica no mercado de combustíveis. Na produção deste combustível, a sacarificação pelo uso de complexo enzimático representa a etapa de maior custo e a fermentação em estado sólido (FES) surge como uma das técnicas mais utilizadas para a produção deste complexo enzimático (LEVER et al., 2010).

O estudo da produção de complexos enzimáticos pelo uso de diferentes microrganismos representa um tipo de pesquisa de interesse para redução de custos 
da produção de etanol de celulose, e a busca por novos microrganismos é uma destas atividades, sendo que o Cerrado representa um dos ambientes de interesse por ter grandes dimensões e variada biodiversidade (MUSSATO et al.,2010).

No presente trabalho, foi feita a avaliação do potencial de um dos fungos do Cerrado na produção de complexo enzimático celulósico pelo uso do fungo Chrysonilia sitophila. A produção de complexos enzimáticos foi realizada com composições de meio sólido distintas em $72 \mathrm{~h}$ de FES e com a melhor composição de meio sólido, foi estudado o efeito do tempo de fermentação gasto na produção do extrato enzimático (FES) na geração de proteínas, crescimento celular da FES e produção de etanol pelo uso do extrato enzimático gerado na FES.

$\mathrm{Na}$ produção de energia por resíduos agroindustriais, tem-se o interesse na produção de etanol de segunda geração, com o uso do bagaço de cana-de-açúcar que representa uma fonte potencial de etanol, pois é um resíduo lignocelulósico abundante no Brasil (SANTOS et al., 2008, PANDEY et al., 2000).

$O$ processo de produção de etanol celulósico de bagaço de cana pode ser descrito em quatro etapas principais de acordo com a Figura 1: pré-tratamento do bagaço de cana para a abertura das fibras, hidrólise enzimática, fermentação e destilação.

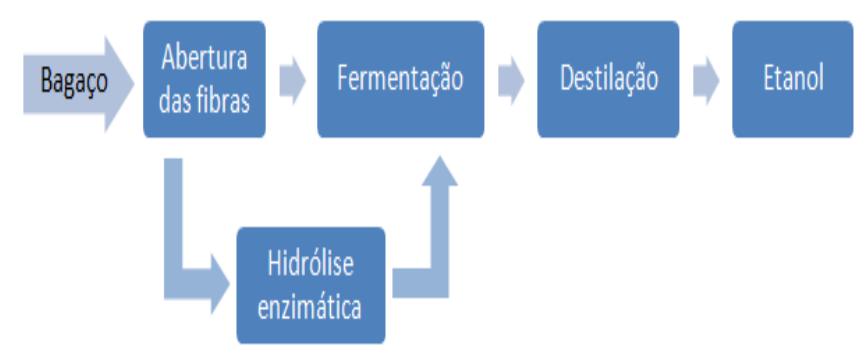

Figura 1. Principais processos utilizados para a produção de etanol celulósico.

A etapa de abertura das fibras pode ser realizada de diversas formas como, por exemplo, pela explosão a vapor, na qual vapor de água em condições de temperatura e pressão gera a ruptura do material lignocelulósico em celulose, hemicelulose e lignina como mostrado a Figura 2.

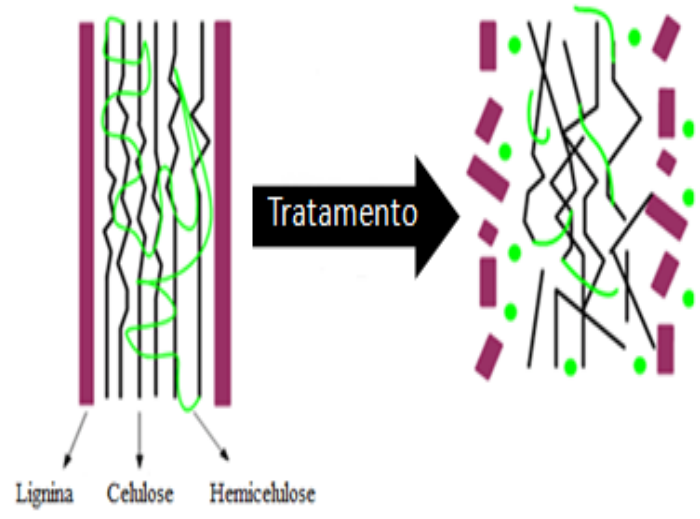

Figura 2. Representação do complexo lignocelulósico antes e depois de um tratamento para abertura das fibras.

$\mathrm{Na}$ produção de etanol de segunda geração, concentrações de etanol variáveis entre $12 \mathrm{~g} / \mathrm{L}$ e $21 \mathrm{~g} / \mathrm{L}$ são citadas na literatura (SUKUMARAN et al, 2009; LEVER et al, 2010), sendo que os desafios deste processo são o aumento da concentração de etanol e redução do custo operacional, principalmente na etapa de hidrólise, a qual vem sendo estudada de diferentes formas na busca de novos microrganismos e evolução da produção de enzima por fermentação em estado sólido (FES) como etapa adicional do processo fermentativo (Figura 3).

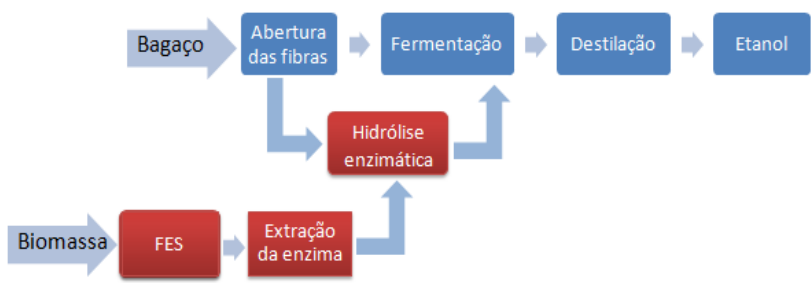

Figura 3. Principais processos utilizados para a produção de etanol celulósico com destaque para a hidrólise enzimática (Fonte: autores).

No presente trabalho, foram avaliadas tanto a etapa de geração de enzima quanto a etapa de produção de etanol, utilizando complexo de enzima de fungo do Cerrado e bagaço de cana-de-açúcar tratado por explosão a vapor. 


\section{MATERIAIS E MÉTODOS}

\section{Microrganismos}

Na produção do extrato enzimático, foi utilizado o fungo do Cerrado da região do Triângulo Mineiro/MG Chrysonilia sitophila, que foi isolado pelos autores pela técnica de esgotamento em placa de petri, com semeadura feita estria e identificação por características morfológicas no Laboratório Exame SA (Uberlândia, MG). O mesmo era preservado em tubos de ensaio, contendo meio ágar Czapek incubado em estufa $\left(25^{\circ} \mathrm{C} \pm 1^{\circ} \mathrm{C}\right)$ por 5 dias para o crescimento e posteriormente, conservado sob refrigeração com renovação de cultivo a cada 20 dias. $\mathrm{Na}$ produção de etanol foi utilizada a Saccharomyces cerevisiae Y904 (Sc) na forma liofilizada, que era ressuspensa em extrato enzimático da FES nas fermentações alcoólicas.

\section{Fermentação em estado sólido (FES)}

Foram realizadas fermentações em estado sólido para produção de extrato enzimático destinado a hidrólise da celulose em açúcares para uso nas fermentações alcoólicas (itens 2.3 e 2.4). Em cada FES os meios sólidos foram inoculados com $10^{5}$ a $10^{6}$ esporos/g de Chrysonilia sitophila suspenso em água. Os esporos foram obtidos por centrifugação de células geradas por fermentação em meio Czapek líquido $\left(30^{\circ} \mathrm{C}\right.$, 48h), e a FES foi realizada em erlenmeyers de $500 \mathrm{~mL}$ contendo $50 \mathrm{~g}$ de meio sólido com $51 \%$ de umidade, $34 \mathrm{~mL}$ de soro de leite na concentração $60 \mathrm{~g} / \mathrm{L}$ e $34 \mathrm{~mL}$ do inóculo.

As FES foram realizadas em dois conjuntos de ensaios destinados à produção de extratos enzimáticos de sacarificação. No primeiro, fermentações em estado sólido foram conduzidas por 72 horas, utilizando 12 composições de meio sólido distintas, como mostrado a Tabela 1 , nas quais foi avaliada a concentração de etanol ao final da fermentação alcóolica. No segundo conjunto de ensaios, avaliou-se o efeito do tempo de FES na qualidade do extrato enzimático, com a utilização de composição de meio de FES fixo correspondente ao meio sólido que gerou o melhor extrato enzimático do primeiro conjunto de ensaios.

Tabela 1 - Composição do meio de fermentação em estado sólido (proporção em volume)

\begin{tabular}{|c|c|c|c|}
\hline Meio & $\begin{array}{c}\text { Bagaço } \\
\text { de cana } \\
\text { tratado }\end{array}$ & $\begin{array}{c}\text { Farelo } \\
\text { de } \\
\text { arroz }\end{array}$ & $\begin{array}{c}\text { Palha } \\
\text { de } \\
\text { arroz }\end{array}$ \\
\hline 1 & $0 \%$ & $50 \%$ & $50 \%$ \\
\hline 2 & $0 \%$ & $33 \%$ & $67 \%$ \\
\hline 3 & $0 \%$ & $67 \%$ & $33 \%$ \\
\hline 4 & $50 \%$ & $50 \%$ & $0 \%$ \\
\hline 5 & $67 \%$ & $33 \%$ & $0 \%$ \\
\hline 6 & $33 \%$ & $67 \%$ & $0 \%$ \\
\hline 7 & $50 \%$ & $0 \%$ & $50 \%$ \\
\hline 8 & $33 \%$ & $0 \%$ & $67 \%$ \\
\hline 9 & $67 \%$ & $0 \%$ & $33 \%$ \\
\hline 10 & $33 \%$ & $33 \%$ & $33 \%$ \\
\hline 11 & $100 \%$ & $0 \%$ & $0 \%$ \\
\hline 12 & $0 \%$ & $100 \%$ & $0 \%$ \\
\hline
\end{tabular}

\section{Produção de etanol}

O substrato utilizado para a produção de etanol foi composto de bagaço de cana in natura e bagaço de cana tratado por explosão de vapor, pelo método de Cromatografia de Alta Eficiência (HPLC). Foram usados 200 $\mathrm{g} / \mathrm{L}$ de bagaço (16 $\mathrm{g}$ de bagaço de cana tratado e $4 \mathrm{~g}$ de bagaço in natura para $100 \mathrm{~mL}$ de extrato enzimático) em fermentações em erlenmeyers, sobre mesa agitada com volume total de $250 \mathrm{~mL}$. Todas as fermentações de produção de etanol, foram realizadas na temperatura de $28 \pm 2^{\circ} \mathrm{C}$, concentração de inóculo de $30 \mathrm{~g} / \mathrm{L}$ e tempo de fermentação de $48 \mathrm{~h}$.

\section{RESULTADOS E DISCUSSÃO}

Foram apresentadas na Figura 3 as concentrações de etanol associadas à hidrólise do bagaço, pois testes de fermentação alcóolica do extrato enzimático puro, não apresentados neste trabalho, mostram que não há formação de etanol associada à fermentação do extrato enzimático. Pode-se notar que o meio M6, composto de 33\% de bagaço de cana tratado e $67 \%$ de farelo de arroz foi o que favoreceu a maior conversão de bagaço em etanol com geração de aproximadamente 5,3 
$\mathrm{g} / \mathrm{L}$ de etanol. Assim este meio foi utilizado nos estudos seguintes.

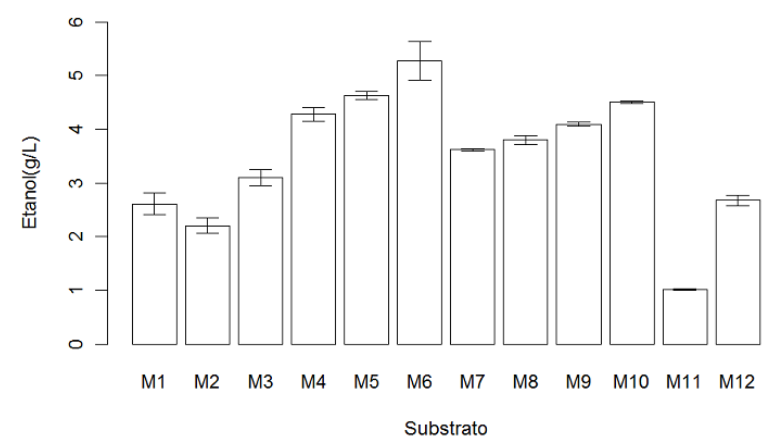

Figura 3 - Efeito da composição do meio de FES na produção etanol

A Figura 4 é mostrada a avaliação da produção de etanol na melhor composição de meio, formulação M6, para diferentes tempos de FES. Pode-se observar que, entre os tempos testados, o de maior concentração de etanol foi o de 72 horas de fermentação em estado sólido.

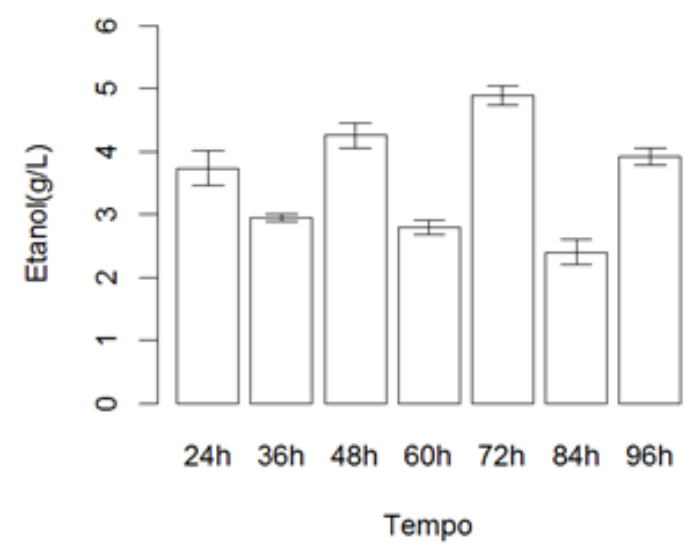

Figura 4 - Concentração de etanol para diferentes tempos de FES

O efeito da carga do reator e percentagem de bagaço, para o melhor tempo de desempenho (72h) é apresentado na figura 5 que mostra que, tanto a carga quanto a porcentagem de bagaço de cana-de-açúcar são variáveis importantes na fermentação alcoólica, sendo que os melhores resultados foram, para a situação de menor carga e menor porcentagem de bagaço. Outro fato observado é que a produção de etanol, utilizando o fungo do Cerrado, foi expressiva quando comparada com a literatura. Sukumaran e colaboradores, por exemplo, em estudo semelhante obtiveram $12,3 \mathrm{~g} / \mathrm{L}$ de etanol.

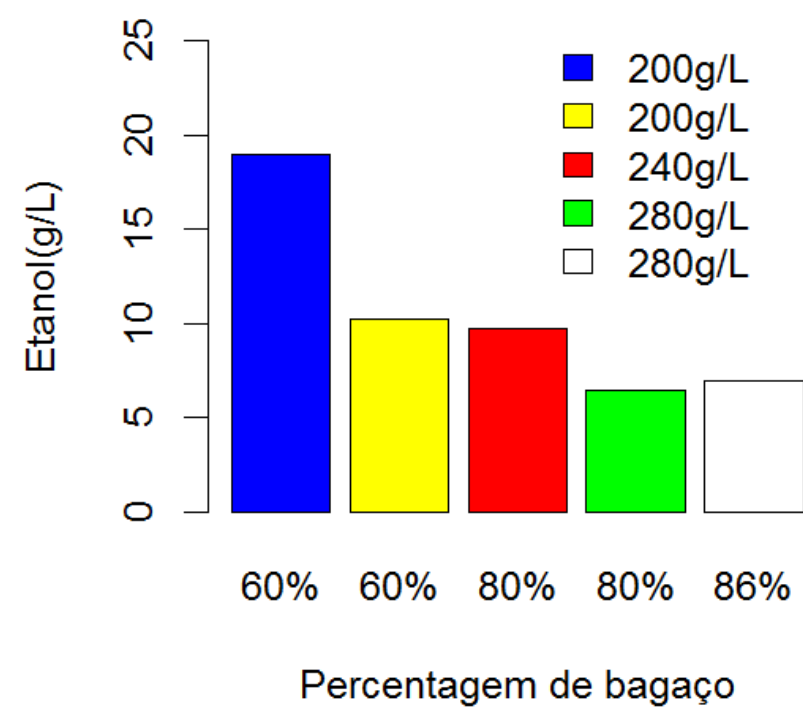

Figura 5- Concentração de etanol para diferentes cargas de bagaço de cana-de-açúcar e inerte no reator.

\section{CONCLUSÃO}

Os resultados indicaram a potencialidade do emprego do fungo Chrysonilia sitophila para degradação da celulose. A FES apresentou os melhores resultados quando conduzida em 72 horas com substrato composto por $33 \%$ de bagaço de cana tratado com explosão a vapor e $67 \%$ de farelo de arroz.

A produção de etanol utilizando fungo do Cerrado nas condições estudadas foi satisfatória e é influenciada tanto pela carga de bagaço de cana-de-açúcar quanto pela proporção do mesmo.

\section{REFERÊNCIAS BIBLIOGRÁFICAS}

FISCHER, J.; LOPES, V. S.; RAMADAN, L.; FERREIRA, J. V. G.; GALVÃO, C. M. A.; TEODORO, J. C.; FILHO, U. C.; CARDOSO, V. L. Produção de extrato enzimático e etanol celulósico. $7^{\mathrm{o}}$ Congresso Internacional de Bioenergia, São Paulo - SP, 2012.

FISCHER, J.; SILVA, N. K. G.; ROCHA, N. R. A. F.; FILHO, U. C.; CARDOSO, V. L. 
Avaliação do potencial de fungos do Cerrado na produção de celulase: um estudo bidimensional. In: SIMPÓSIO NACIONAL DE BIOPROCESSOS, 18., 2011, Caxias do Sul. Anais... Caxias do Sul, 2011.

LEVER, M; HO, CORD, G.; F RUWISCH, R. Ethanol from lignocellulose using crude unprocessed cellulase from solid-state fermentation Bioresource Technology, v. 101, p.7083-7087, 2010 .

LEVER, M.; HO, G.; CORD-RUWISCH, R. Ethanol from lignocellulose using crude unprocessed cellulose from solid-state fermentation. Bioresource Technology, n. 101, p. 7083-7087, 2010.

MOSIER, N.; WYMAN, C.; DALE, B.; ELANDER, R.; LEE, Y. Y.; HOLTZAPPLE, M.; LADISCH, M. R. Features of promising technologies for pretreatment of lignocellulosic biomass. Bioresource Technology, n. 96, p. 673-686, 2005.

SUKUMARAN, R., K.; SINGHANIA, R. R.; MATHEW, G. M.; PANDEY, A. Cellulase production using biomass feed stock an its application in lignocelluloses saccharification for bio-ethanol production. Renewable Energy, n. 34, p. 421-424, 2009.

\section{AGRADECIMENTOS}

Os autores agradecem a CAPES, CNPq e FAPEMIG pelo apoio financeiro, e ao CTC pela doação do bagaço de cana-de-açúcar. 